\title{
Tracking Bifurcating Solutions of a Model Biological Pattern Generator
}

\author{
K. H. WINTERS
}

Theoretical Studies Department, Harwell Laboratory, Didcot OX11 ORA, United Kingdom

\author{
M. R. MYERSCOUGH
}

School of Mathematics and Statistics, University of Sydney, New South Wales 2006, Australia

\author{
P. K. MAINI
}

Department of Mathematics, University of Utah, Salt Lake City, Utah 84112, and Centre for Mathematical Biology, Mathematical Institute, 24-29 St. Giles, Oxford OXI 3LB, United Kingdom

\begin{abstract}
AND
J. D. MURRAY

Centre for Mathematical Biology, Mathematical Institute, 24-29 St. Giles, Oxford OX1 3LB, United Kingdom, and Applied Mathematics, University of Washington,

FS-20, Seattle, Washington 98195
\end{abstract}

Received November 5, 1990

K. H. Winters, M. R. Myerscough, P. K. Maini, and J. D. Murray, Tracking Bifurcating Solutions of a Model Biological Pattern Generator, IMPACT of Computing in Science and Engineering 2, 355-371 (1990).

We study heterogeneous steady-state solutions of a cell-chemotaxis model for generating biological spatial patterns in two-dimensional domains with zero flux boundary conditions. We use the finite-element package ENTWIFE to investigate bifurcation from the uniform solution as the chemotactic parameter varies and as the domain scale and geometry change. We show that this simple cell-chemotaxis model can produce a remarkably wide and surprising range of complex spatial patterns. @1 1990 Academic Press, Inc. 


\section{BIOLOGICAL MOTIVAIION AND THE IMPORTANCE OF PATTERN FORMATION}

The generation of biological pattern and form are key issues in the early development of an embryo. Embryonic cells divide, migrate, and differentiate to form the various organs in the body. Many of these structures have a regular pattern, such as feather and scale patterns, while others have distinctive quasi-regular patterns such as the stripes on alligators and the spots on leopards. These markings are usually formed early in development. For example, in the case of the stripes on a zebra the pattern is probably laid down by the fourth week; this compares with a 365-day gestation period. Modeling of biological pattern and form is discussed in detail in the book by Murray [1]. The specific model discussed here was proposed in [2] and various biological applications are presented in [3].

Pigment patterns are generated by cells which make melanin; these cells lie in the skin layers made up of the epidermis and the dermis. Cell migration and cell differentiation are two basic processes involved in the generation of pigmentation patterns of the vertebrate integument. Several models have been proposed for skin patterning. For example, reaction-diffusion models [4-7, 1] hypothesize the existence of chemicals (morphogens) which react and diffuse and, under appropriate conditions, generate spatially heterogeneous patterns. Cells are then assumed to be preprogrammed to differentiate according to the level of the chemical they experience. Much of the justification for such models is still circumstantial. The Oster-Murray mechanochemical theory (for a review see $[8,1]$ ) of biological pattern formation can also generate patterns similar to those created by reaction-diffusion models and their solutions exhibit similar developmental geometric constraints first described in the context of skin patterns by Murray [6]. The concept of developmental constraints are discussed in detail in [2]. In the model we discuss here the patterns are in cell density and the development of the patterns involves movement of actual biological cells which we believe to be the precursors of melanophores.

The model proposed by Oster and Murray [2] takes account of cell motility and chemotaxis, the chemical processes by which cells migrate up a chemical gradient. In their model the cells both respond to and produce the chemoattractant. This model was recently proposed for the stripe and shadow patterns on the alligator (Alligator mississipiensis) $[9,10]$. Experimental results unequivocably show that scale is crucial in determining the number of stripes on alligators, and that higher densities of melanin-forming cells are found in the dark stripe regions of the alligator.

In Section 2 we briefly describe the chemotaxis model, while in Section 3 we describe in detail the numerical methods involved in using ENTWIFE to analyze the differential equation system. In Section 4 we present a selection of spatially patterned solutions and discuss the complex bifurcation picture 
that was found. We also describe the effect on the patterns of domain growth since in many developmental situations spatial organization of pattern takes place on a time scale commensurate with significant growth of the embryo $[10]$.

\section{Cell-Chemotaxis Model Equations}

Chemotaxis is a major factor in many developmental situations and this is the key aggregation force in our model. The model, discussed in detail in $[2,3]$, involves two dependent variables, the cell density, $n(\mathbf{r}, t)$, and the chemoattractant concentration, $c(\mathbf{r}, t)$, where $\mathbf{r}$ and $t$ are the spatial coordinate and time, respectively. The model consists of a pair of coupled nonlinear partial differential equations which describes the motion of the cells and the production, diffusion, and degradation of the chemoattractant.

The equation for cell density is

$$
\begin{aligned}
& \frac{\partial n}{\partial t}=D_{n} \nabla^{2} n-\alpha \nabla \cdot(n \nabla c)+r n(N-n), \\
& \text { diffusion chemotaxis cell growth }
\end{aligned}
$$

where we have assumed that cell division may be described by a simple logistic growth where $r N$ is the linear mitotic growth rate. The chemotaxis term reflects the fact that cells move up a concentration gradient in $c$; the chemotaxis parameter is $\alpha$. We also assume that the cells diffuse with diffusion coefficient $D_{n}$. The parameters $r, N, \alpha$, and $D_{n}$ are all positive constants.

The cells are assumed to secrete their own chemoattractant in a MichaelisMenten fashion. The chemoattractant diffuses with diffusion coefficient $D_{c}$ and degrades according to first-order kinetics. The equation for the chemotactic concentration $c$ then takes the form

$$
\frac{\partial c}{\partial t}=D_{c} \nabla^{2} c+\frac{S n}{\beta+n}-\gamma c
$$

where $S, \beta, \gamma$, and $D_{c}$ are all positive constants.

We are interested in pattern formation on finite two-dimensional domainsthe model for the skin of developing vertebrates-so we consider these equations on a finite domain $D$ with zero flux boundary conditions, namely

$$
\mathbf{n} \cdot \nabla c(\mathbf{r})=\mathbf{n} \cdot \nabla n(\mathbf{r})=0 \quad \text { for } \quad \mathbf{r} \in \partial D,
$$


where $\mathbf{n}$ is the unit outward normal to the boundary $\partial D$. The mathematical problem consists of Eqs. (2.1) and (2.2), with boundary conditions (2.3).

To solve the equations numerically we cast the system in nondimensional terms by writing

$$
\begin{aligned}
& \mathbf{r}^{*}=\left[\frac{\gamma}{D_{c} s}\right]^{1 / 2} \mathbf{r}, \quad t^{*}=\frac{\gamma t}{s}, \quad n^{*}=\frac{n}{\beta}, \quad c^{*}=\frac{\gamma c}{S}, \\
& N^{*}=\frac{N}{\beta}, \quad D^{*}=\frac{D_{n}}{D_{c}}, \quad \alpha^{*}=\frac{\alpha S}{\gamma D_{c}}, \quad r^{*}=\frac{r \beta}{\gamma}
\end{aligned}
$$

where $s$ is a scale factor. We could of course absorb $s$ into a new scaling for $t$ and $\mathbf{r}$ but we retain it here since we can think of $s=1$ as the unit domain and carry out the computation on a fixed domain size and increase $s$ to simulate larger domains, a procedure used in the simulation of reactiondiffusion systems [4-6]. With (2.4) the nondimensional equations become, on omitting the asterisks for notational simplicity,

$$
\begin{gathered}
\frac{\partial n}{\partial t}=D \nabla^{2} n-\alpha \nabla \cdot(n \nabla c)+s r n(N-n) \\
\frac{\partial c}{\partial t}=\nabla^{2} c+s\left[\frac{n}{1+n}-c\right] \\
\mathbf{n} \cdot \nabla c(\mathbf{r})=\mathbf{n} \cdot \nabla n(\mathbf{r})=0, \quad \mathbf{r} \in \partial D,
\end{gathered}
$$

where $\partial D$ is now the boundary of the scaled domain. To be specific we consider the domain $D$ to be rectangular with width $L_{x}$ and length $L_{y}$; we use Cartesian coordinates $(x, y)$.

The system (2.5) has two uniform steady states, $n=0, c=0$ and $n=N$, $c=N /(1+N)$, and a linear analysis about these uniform states shows that the trivial steady state is always unstable but that the nontrivial spatially homogeneous solution can be driven unstable in certain parametcr regimes by spatially heterogeneous perturbations and evolve to inhomogeneous spatial patterns in $n$ and $c$. The form of the pattern is determined primarily by the nondimensional parameter set $(D, \alpha, r, N)$ and the size and shape of the domain. The linearized system has solutions of the form $\cos \left(m \pi x / L_{x}\right)$ $\times \cos \left(l \pi y / L_{y}\right)$ for integers $m$ and $l$. A pattern of lateral stripes, for example, is given by the $(0, l)$ mode where the higher the value of $l$ the more stripes we have on the domain. In order to select a mode $(m, l)$ on a rectangular domain we require (see Appendix for details) 


$$
\begin{gathered}
\frac{r N s^{2}}{D}=\pi^{4}\left\{\frac{m^{2}}{L_{x}^{2}}+\frac{l^{2}}{L_{y}^{2}}\right\}^{2} \\
{\left[r N+D-\frac{N \alpha}{(1+N)^{2}}\right]^{2}=4 r D N .}
\end{gathered}
$$

Thus, our linear analysis predicts that the uniform steady state will evolve to a spatially nonuniform steady state in the appropriate parameter space, the form of the final pattern depending on the parameters. We now proceed to analyze the full nonlinear system for possible steady states.

\section{Numerical Details}

\subsection{Finite-Element Equations}

The equations (2.5a) and (2.5b) are discretized in the finite-element approximation using a standard Galerkin formulation with the second-order terms integrated by parts. This leads to the following form:

$$
\begin{aligned}
& \int_{D} \nabla f_{n} \cdot\left[D \nabla_{n}-\alpha n \nabla c\right] d V-\int_{\partial D} f_{n}[D \nabla n-\alpha n \nabla c] \cdot d \mathbf{S} \\
&-\int_{D} f_{n}\left[\operatorname{srn}(N-n)-\frac{\partial n}{\partial t}\right] d V=0 \\
& \int_{D} \nabla f_{c} \cdot \nabla c d V-\int_{\partial D} f_{c} \nabla c \cdot d \mathbf{S}-\int_{D} f_{c}\left[s\left(\frac{n}{1+n}-c\right)-\frac{\partial c}{\partial t}\right] d V=0 .
\end{aligned}
$$

In these equations, $D$ is the spatial region being discretized, $\partial D$ is the boundary of $D, d \mathbf{S}$ is the vector area with direction out of the domain, $f_{n}$ is a test function for the variable $n$, and $f_{c}$ is a test function for the variable $c$.

The integration by parts gives a boundary integral for each equation over $\partial D$. The integrand consists of a normal flux and the boundary integral contributes only when this flux is nonzero. For the present boundary conditions $(2.5 \mathrm{c})$ all boundary integrals are zero.

In our finite-element approximation of this weak formulation of the full system we expand the cell density and chemoattractant in quadratic functions based on nine-noded quadrilateral elements. A Galerkin formulation is adopted in which the test functions $f_{n}$ and $f_{c}$ are chosen from the same set of quadratic basis functions. The resulting integrals of products of quadratic functions are evaluated numerically by Gauss quadrature. The nonlinear algebraic equations for the unknown nodal values of the cell density and chemoattractant are linearized using a Newton-Raphson procedure, and the so- 
lution of the linear set of equations at each iteration is obtained using a direct. frontal solver.

\subsection{Bifurcation Algorithms}

We write the set of nonlinear algebraic equations which result from the finite-element discretization of (2.5) as

$$
\mathbf{M} \frac{\partial \mathbf{x}}{\partial t}+\mathbf{f}(\mathbf{x}, \lambda, \mathbf{p})=0 \quad(\mathbf{x} \in \mathbf{X})
$$

where $\mathbf{f}$ is a smooth nonlinear function, $\mathbf{M}$ is a linear operator on $R^{n}$ where $n$ is the number of degrees of freedom in the discretization, $\lambda$ is a bifurcation parameter, and $\mathbf{p}$ is a vector of control parameters. We distinguish the bifurcation parameter $\lambda$ from the control parameters $p$ because we seek the change of behavior as this particular parameter is varied.

In the present problem we are concerned only with steady-state solutions of (3.3), that is, solutions $\mathbf{x}$ which satisfy

$$
\mathbf{f}(\mathbf{x}, \lambda, \mathbf{p})=\mathbf{0},
$$

and we wish to locate the critical values of $\lambda$ at which bifurcations in the solutions occur. Here, the bifurcation parameter is the chemotactic coefficient $\alpha$ in (2.5) and the control parameters are $r, D, N$, and the scale parameter $s$. Our first objective will be to locate these bifurcations for fixed values of the control parameters. Having located a singular point we then obtain the variation of the solution with the critical bifurcation parameter $\alpha$ with the control parameters fixed. We may also fix $\alpha$ and vary the control parameters. In this way a path of bifurcation points is traced out, and this path may itself have a singular point that we wish to locate.

To understand how we are able to obtain information on possible bifurcations and instabilities from the steady-state equations, we consider the linear stability of a steady solution $\mathbf{x}_{0}$ of Eq. (3.4) with respect to a small perturbation $\mathbf{x}_{1}$. The behavior of the perturbation $\mathbf{x}_{1}$ is governed to lowest order by the linear equation

$$
\mathbf{M} \frac{\partial \mathbf{x}_{1}}{\partial t}+\mathbf{f}_{x}\left(\mathbf{x}_{0}, \lambda, \mathbf{p}\right) \mathbf{x}_{1}=0 .
$$

Now let $\mathscr{X}$ be a generalized eigenvector of $\mathbf{f}_{x}\left(\mathbf{x}_{0}, \lambda, \mathbf{p}\right)$ with eigenvalue $\sigma$ such that

$$
\mathbf{f}_{x} \mathscr{X}=\sigma \mathbf{M} \mathscr{X}
$$


Then if the perturbation $x_{1}$ is along $\mathscr{X}$ it behaves as

$$
\mathbf{x}_{1}(t)=\epsilon \exp [-\sigma t] \mathscr{X}
$$

where $\epsilon$ is the component of $\mathbf{x}_{1}$ along $\mathscr{X}$ at $t=0$. The steady solution $\mathbf{x}_{0}$ is linearly stable if all the generalized eigenvalues $\sigma$ have $\operatorname{Re}(\sigma)>0$; all small perturbations will decay until the steady state is restored. We note that since $f_{x}$ is real the eigenvalues in (3.6) are either real or else occur in complex conjugate pairs.

As the bifurcation parameter $\lambda$ varies, the linear stability of the steady solution $\mathbf{x}_{0}$ changes when one or more of the eigenvalues in (3.6) crosses the imaginary axis. Thus there is a critical value of $\lambda$ for which $\sigma=0$ and $\mathbf{f}_{x}$ is singular; this critical value is called a singular or bifurcation point of Eq. (3.4). We know from the Implicit Function Theorem that the uniqueness of the steady solution $\mathbf{x}_{0}$ cannot be guaranteed when the Jacobian $\mathbf{f}_{x}$ becomes singular and this usually (but not necessarily) marks the appearance of multiple steady-state solutions.

We note that if a generalized eigenvalue becomes purely imaginary, that is, $\sigma= \pm i \omega$, then Eq. (3.4) has a Hopf bifurcation which gives rise to periodic solutions of angular frequency $\omega$ at a critical value of $\lambda$, although the Jacobian $\mathbf{f}_{x}$ is not actually singular at that point. We do not address the existence of Hopf bifurcations in the present paper.

3.2.1. Stability of Solution Branches. From the above discussion it might appear that the stability of solution branches can be deduced only from a computation of the full eigenvalue spectrum of $\mathbf{f}_{x}$. Fortunately, limited information on stability can be inferred from the Jacobian determinant: since this is the product of the eigenvalues then its sign must equal $(-1)^{q}$, where $q$ is the number of negative, unstable eigenvalues. Thus, a negative sign indicates that the branch is unstable since there is at least one negative eigenvalue, but a positive sign does not imply stability since there might be an even number of unstable eigenvalues.

The sign of the Jacobian determinant is also useful for detecting bifurcation points along a solution branch while varying the bifurcation parameter $\lambda$, since the sign will in general change when a singular point is passed.

3.2.2. Extended Systems. The general procedure we adopt for locating bifurcation points of Eq. (3.4) is to solve the equation simultaneously with conditions satisfied at the bifurcation. One condition which must always hold, by definition, is that the Jacobian matrix is singular so that it has at least one zero eigenvalue. Additional conditions may hold depending on the type of bifurcation which is to be located and these conditions can be derived from singularity theory [11]. The resulting extended system of equations are solved by Newton's method to give both the solution at the bifurcation point and 
the value of the bifurcation parameter. This approach is described in detail in [12].

The different types of singular points with which we are concerned in the present problem are:

primary bifurcations from a trivial solution (that is, one which does not depend on the bifurcation parameter);

simple turning points on nonlinear solution branches.

In addition, we detected the presence of secondary bifurcations from the nonlinear branches that originate at the primary bifurcations from the trivial solution, but we did not attempt to locate their positions exactly or to step onto the secondary bifurcating branches.

In the present problem the spatial patterns correspond to nonlinear branches that bifurcate from the uniform trivial solution. We define a trivial solution of Eq. (3.4) to be a solution which is independent of $\lambda$ and denote it $x_{0}$. We locate bifurcations from $\mathbf{x}_{0}$ by computing the eigenvectors $\xi$ of the Jacobian matrix $\mathbf{f}_{x}\left(\mathbf{x}_{0}, \lambda, \mathbf{p}\right)$ which have a simple zero eigenvalue; that is, we solve

$$
\begin{gathered}
\mathbf{f}_{x}\left(\mathbf{x}_{0}, \lambda, \mathbf{p}\right) \xi=0 \\
l(\xi)-1=0,
\end{gathered}
$$

where the last equation defines a normalization for the right eigenvector $\xi$.

The branch of solutions bifurcating away from the singular point $\left(\mathbf{x}_{0}, \lambda\right.$, p) may be approximated by

$$
\begin{gathered}
\mathbf{x}=\mathbf{x}_{0}+\epsilon \xi_{0}+\cdots, \\
\lambda=\lambda_{0}+1 / 2 \epsilon^{2} \delta+\cdots,
\end{gathered}
$$

where $\delta$ is defined by a complex expression given by [13].

The above expressions reflect the fact that the primary bifurcations are pitchfork in shape. They will be either sub- or supercritical according to whether $\delta$ is negative or positive. In order to compute the solution on any nonlinear branch which bifurcates from the trivial solution we use Eqs. (3.9) and (3.10) to construct an initial guess at the new value of $\lambda$; Newton's method will then converge quadratically to the solution on the branch for sufficiently small $\left(\lambda-\lambda_{0}\right)$. The branch is then followed for varying $\lambda$ by standard continuation techniques (see Section 3.2.3).

To locate limit points (also called turning points or one-sided bifurcation points) we use the extended system proposed by [14]; 


$$
\begin{gathered}
\mathbf{f}(\mathbf{x}, \lambda, \mathbf{p})=0 \\
\mathbf{f}_{x}(\mathbf{x}, \lambda, \mathbf{p}) \xi=0 \\
l(\xi)-1=0,
\end{gathered}
$$

where the last equation in (3.11) is a normalization condition, as in (3.8).

3.2.3. Continuation. Euler-Newton continuation is an effective means of following a particular solution branch. In its simplest form the solution $\mathbf{x}_{0}$ obtained at $\lambda=\lambda_{0}$ and its derivative $\partial \mathrm{x}_{0} / \partial \lambda$ are used to predict the solution $\mathbf{x}_{1}$ at a new value $\lambda_{1}$, from

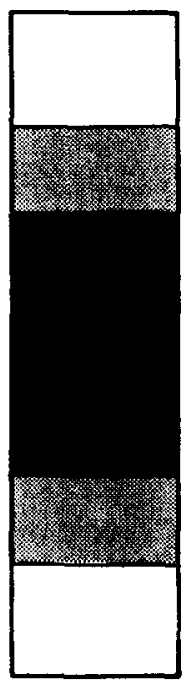

(a)

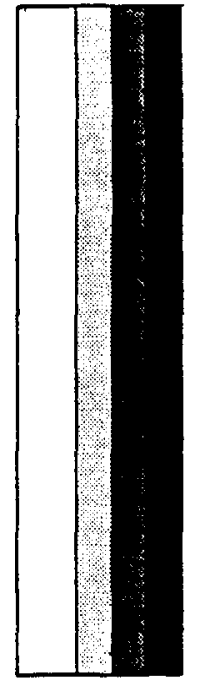

(b)

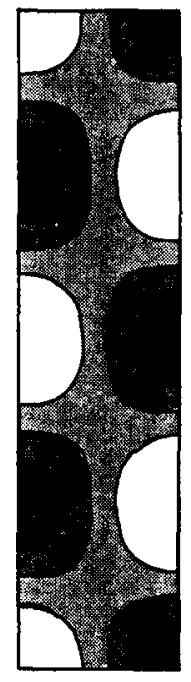

(c)

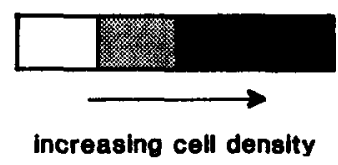

FIG. 1. The parameters $(D, r, N)$ and the size and shape of the domain were fixed, and $\alpha$ was used as the bifurcation parameter. (a) $D=0.25, r=1.52, N=1, s=1$. Bifurcation to $(0,2)$ mode at $\alpha=12.01$. (b) $D=0.25, r=24.35, N=1, s=1$. Bifurcation to $(1,0)$ mode at $\alpha$ $=118.54$. In this case mode $(0,4)$ is also a solution. To separate these degenerate modes we solved the system on a $1 \times 4.1$ domain. (c) $D=0.25, r=1.52, N=1, s=1$. Bifurcation to ( 1 , 4) mode at $\alpha=27.06$. (In all the above cases we only show the steady-state cell density. The chemoattractant concentration is qualitatively similar.) 

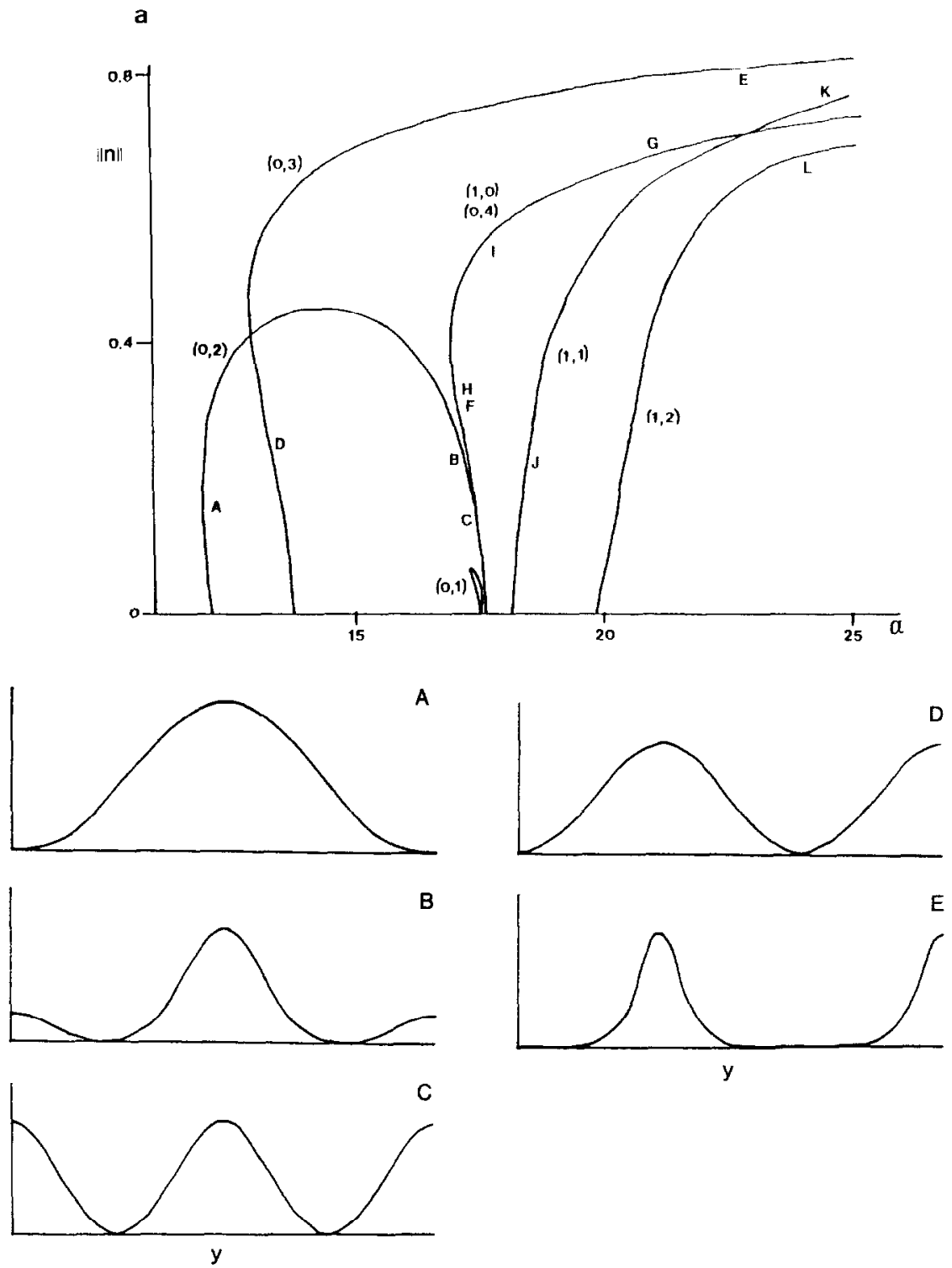

$\mathrm{C}$

Fig. 2. (a) Bifurcation diagram for fixed parameter values $r=1.52, N=1, s=1, D=0.25$ and varying $\alpha$ on a $1 \times 4$ domain. This diagram only shows the upper branches of primary bifurcations; secondary bifurcations from these branches were observed but not studied in detail. The letters $A$ to $L$ on the state diagram denote the patterns indicated. Note that modes $(0,4)$ and $(1,0)$ are degenerate. Note also that the points of intersection of branches are not bifurcation points but merely where the branches overlap. (b) A different choice of measure shows the nature of the bifurcation of mode $(0,1)$ more clearly. The letters $P$ to $U$ denote the patterns indicated. $\left[\|n\|=\int_{D}|N-n| d x d y / \int_{D} d x d y, D=\right.$ domain: $n(0,0)$ means the value of $n$ at the point $(0,0)$.] 

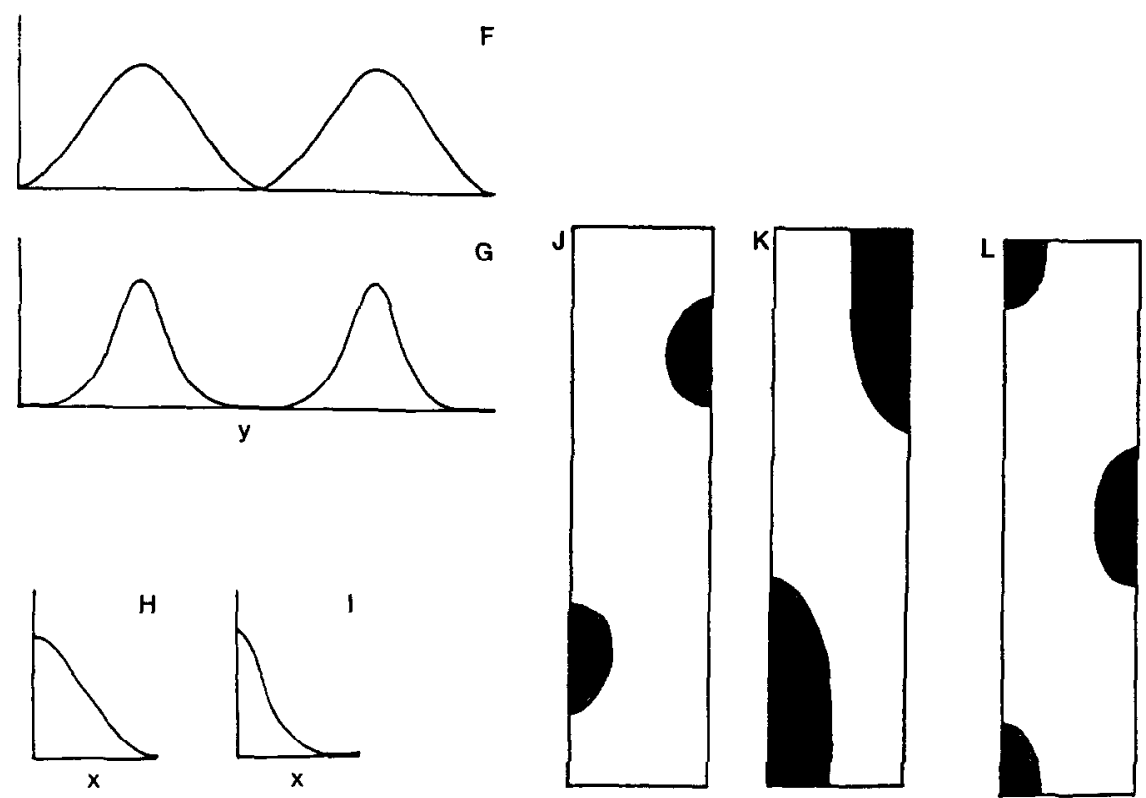

FIG. 2-Continued

$$
\mathbf{x}_{1}=\mathbf{x}_{0}+\frac{\partial \mathbf{x}_{0}}{\partial \lambda}\left(\lambda_{1}-\lambda_{0}\right)
$$

The Newton-Raphson iterations converge rapidly at each value of $\lambda_{j}$, for a suitable step size $\left(\lambda_{j}-\lambda_{j-1}\right)$, but this procedure ultimately fails at a limit point in the solution curve, where the Jacobian matrix is singular.

In practice, we used a better method which introduces a pseudo-arclength parameter, $s$ (not to be confused with the scale parameter in Section 2), to parametrize the solution [15]. For continuation in $\lambda=\lambda(s)$ we solve the extended system

$$
\mathbf{f}(\mathbf{x}, \lambda, \mathbf{p})=\mathbf{0}, \quad \mathbf{N}(\mathbf{x}, \lambda, s)=0,
$$

where

$\mathbf{N}=\left[\frac{\partial \mathbf{x}}{\partial s}\left(s_{0}\right)\right]^{T}\left[\mathbf{x}(s)-\mathbf{x}\left(s_{0}\right)\right]+\frac{\partial \lambda}{\partial s}\left(s_{0}\right)\left[\lambda(s)-\lambda\left(s_{0}\right)\right]-\left(s-s_{0}\right)$

With Euler-Newton continuation in $s$ rather than $\lambda$, it is possible to follow the solution around a limit point, since the Jacobian matrix of system (3.13)- 

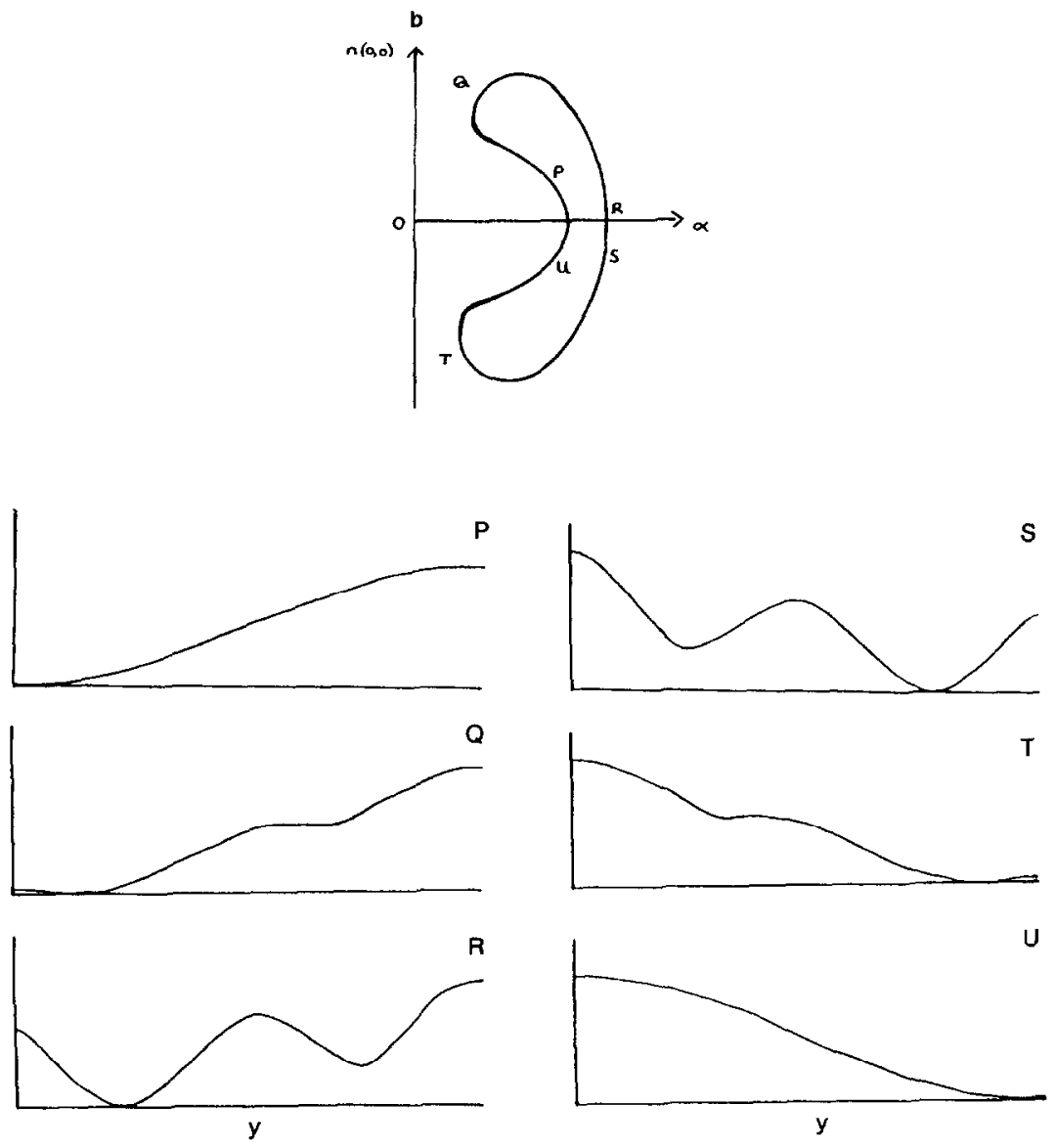

Fig. 2-Continued

(3.14) is nonsingular. The presence of a symmetry-breaking bifurcation point or limit point is determined by monitoring the Jacobian determinant, which changes sign as the singular point is passed.

\section{Spatial Patterns}

In this section we present a selection of results from the application of ENTWIFE to the steady-state problem for $(2.5)$ on a $1 \times 4$ rectangular domain, by way of illustration of certain bifurcation tracking features of ENTWIFE.

(1) Varying one parameter, while keeping all others fixed. In this case we fix the parameter set $(D, r, N)$ and the size and shape of the domain. We use 
the coefficient of chemotaxis, $\alpha$, as the bifurcation parameter. ENTWIFE locates values of $\alpha$ at which bifurcation from the uniform steady state occurs and calculates the nonuniform steady state at these points. Figure 1 illustrates a selection of results from such calculations and Fig. 2 shows a bifurcation diagram obtained in this way.

(2) Varying a parameter along a bifurcation branch. Figures 2 and 3 show the change in the steady-state pattern as $\alpha$ varies along a bifurcation branch.

(3) Varying two parameters. Figure 4 illustrates part of the bifurcation diagram in which a branch is calculated by varying one parameter. It is then possible to step off this branch by varying another parameter.

(4) Varying scale and geometry. Figure 5 shows the effects on the steadystate pattern of changes in scale and geometry.

\section{DisCUSSION}

In this paper we have illustrated the application of the finite-element package ENTWIFE to the study of the steady-state bifurcation behavior of a cellchemotactic model for biological pattern generation. This simple model exhibits a surprisingly complex and diverse range of patterns. In this paper we have presented a selection of steady-state spatial patterns. The paper by Maini

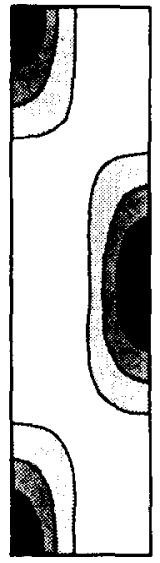

(i)

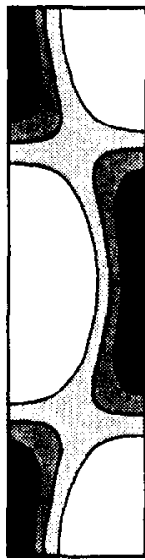

(ii)

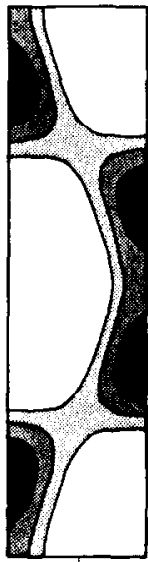

(iii)

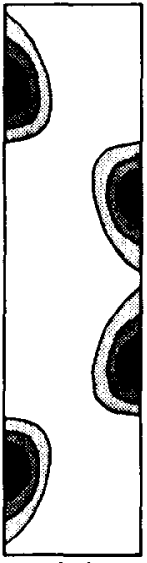

(iv)

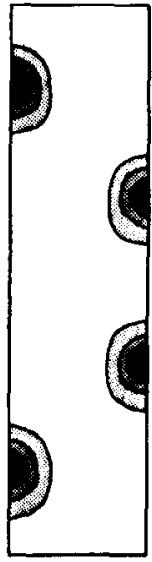

(v)

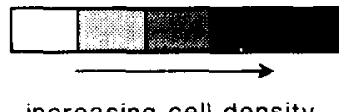

increasing cell density

FIG. 3. Steady-state cell density as $\alpha$ increases along the branch bifurcating at mode $(1,2)$ in Fig. 2. (i) $\alpha=23.75$; (ii) $\alpha=27.59$; (iii) $\alpha=32.71$; (iv) $\alpha=42.95$; (v) $\alpha=63.43$. 


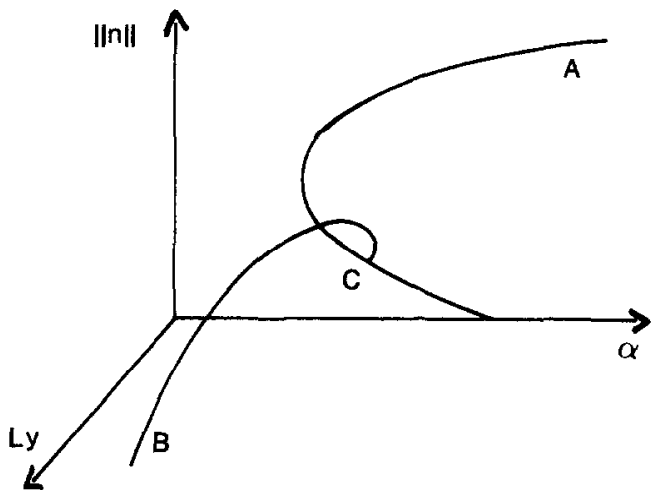

FIG. 4. Bifurcation branches in two-dimensional parameter space. A, bifurcation branch in the $\alpha-\|n\|$ plane; B, bifurcation branch in the $L_{y}-\|n\|$ plane obtained by stepping off A at point C.

et al. [3] contains a wider range of patterns, together with a more complete discussion of the biological significance of these calculations.

The numerical package ENTWIFE was used to calculate points in parameter space where bifurcation occurs from the uniform steady state. The calculations in the Appendix show that it is possible to obtain some of these results simply

(i)

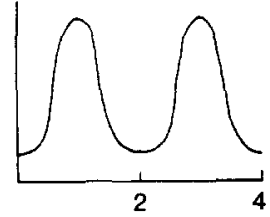

(ii)

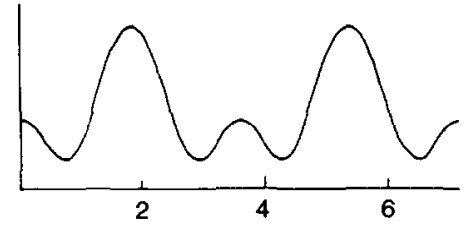

(iii)

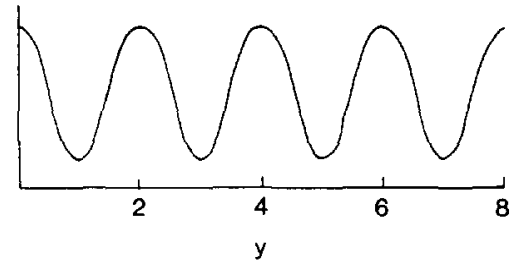

b

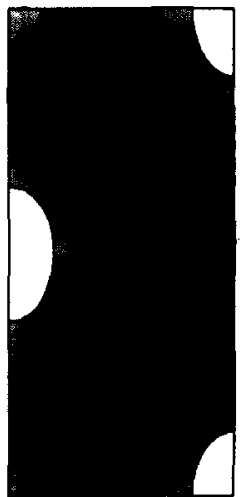

(i)

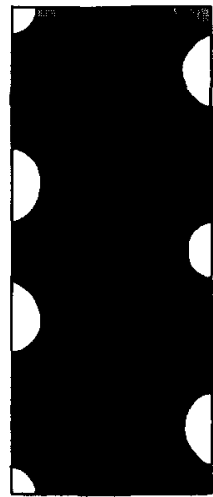

(ii)

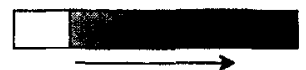

FIG. 5. (a) Schematic diagram of the cross section in the $y$ direction of cell density patterns as $L_{y}$ increases. (i) $L_{y}=4$; (ii) $L_{y}=7.6$; (iii) $L_{y}=8$. (b) Effect of changing domain width. (i) $L_{x}$ $=1.84$; (ii) $L_{x}=1.74$. 
from a linear analysis. However, ENTWIFE can calculate the structure of the steady state and its nonlinear behavior as parameters change along the bifurcation branch. It also enabled us to study the effects on the pattern of changes in scale and geometry. The analysis of the effects of changes in such geometrical parameters is very important in biology.

Here we have illustrated only some of the features of ENTWIFE. Further capabilities and a description of applications in the field of fluid mechanics are given in [16].

In the simulations presented, the stability of the patterns was not investigated. Thus some of the patterns may be unstable. However, in view of the fact that observed developmental patterns may be in response to transient underlying patterns, unstable patterns are still relevant. A detailed eigenvalue analysis along every solution branch would be necessary to prove stability of each pattern and to discount possible Hopf bifurcations to time-periodic pattern. Such a detailed study is possible with the techniques available in ENTWIFE but is outside the scope of the present work.

\section{APPENDIX}

Equations (2.5) exhibit two uniform steady states for $(n, c)$ given by $(0$, $0)$ and $(N, N /(1+N)$. Here we carry out linear stability analysis for the nonzero steady state $(N, N /(1+N))$. (By inspection the steady state $(0,0)$ is always unstable.)

We linearize (2.5) in the usual way by substituting $n=N+u, c=N /(1$ $+N)+v$, where $|u|,|v|$ are small, and retaining only linear terms. This gives the linear system, which governs behavior near the steady state, as

$$
\begin{gathered}
\frac{\partial u}{\partial t}=D \nabla^{2} u-\alpha N \nabla^{2} v-r N s u \\
\frac{\partial v}{\partial t}=\nabla^{2} v+s\left[\frac{u}{(1+N)^{2}}-v\right] \\
\mathbf{n} \cdot \nabla u(\mathbf{x})=\mathbf{n} \cdot \nabla v(\mathbf{x})=0, \quad \mathbf{x} \in \partial D .
\end{gathered}
$$

We look for solutions to (A1) of the form

$$
\left[\begin{array}{l}
u \\
v
\end{array}\right]=\left[\begin{array}{l}
u_{0} \\
v_{0}
\end{array}\right] \exp [i \mathbf{k} \cdot \mathbf{x}+\lambda t],
$$

where $\lambda=\lambda(k)$ determines the temporal growth rate of the periodic spatial disturbance with wave vector $k$. The condition for nontrivial solutions for $u_{0}$ and $v_{0}$ to exist is given by 


$$
\begin{aligned}
\lambda^{2}+\left[(D+1) k^{2}\right. & +r N+s] \lambda \\
& +\left[D k^{4}+\left\{r N s+D s-\frac{(s N \alpha)}{(1+N)^{2}}\right\} k^{2}+r N s^{2}\right]=0
\end{aligned}
$$

and the wave with wavenumber $|\mathbf{k}|$ must satisfy the boundary conditions $(\mathrm{A} 1 \mathrm{c})$. Equation (A2) is the dispersion relation and determines the linear stability of the uniform steady state to disturbances of wave vector $\mathbf{k}$. If $\lambda\left(k^{2}\right)$ $<0$ then the disturbance of wave vector $\mathbf{k}$ will decay with time. If $\lambda\left(k^{2}\right)>0$ for some $k^{2}$ then the disturbance with these wavenumbers will grow and the system will evolve to a nonuniform spatially structured solution.

On a rectangular domain with zero flux boundary conditions the eigenfunctions of the linear system are $\cos \left(m \pi x / L_{x}\right) \cos \left(l \pi y / L_{y}\right)$, where $m$ and $l$ are integers. Thus the appropriate wave vector $\mathbf{k}$ has the form $\left(k_{x}, k_{y}\right)$, where $k_{x}=\left(m \pi / L_{x}\right), k_{y}=\left(I \pi / L_{y}\right)$, and the values of $k^{2}$ which produce a pattern are those where $\lambda\left(k^{2}\right)>0$, where

$$
\mathbf{k} \cdot \mathbf{k}=\mathbf{k}^{2}=\pi^{2}\left(\frac{m^{2}}{L_{x}^{2}}+\frac{l^{2}}{L_{y}^{2}}\right)
$$

At the onset of instability to disturbance with wave vector $\mathbf{k}_{c}, \lambda\left(k_{c}^{2}\right)=0$; that is, $k_{c}$ satisfies

$$
D k^{4}+\left[r N s+D s-\frac{s N \alpha}{(1+N)^{2}}\right] k^{2}+r N s^{2}=0
$$

If we wish to isolate only one unstable wave vector, we require (A4) to have only one solution for $k^{2}$. Forcing (A4) to have equal roots results in the condition

$$
\left[r N s+D s-\frac{s N \alpha}{(1+N)^{2}}\right]^{2}-4 D r N s^{2}=0
$$

From (A4) and (A5) we can deduce that the modulus of the critical wave vector is given by

$$
k_{c}^{2}=s\left(\frac{r N}{D}\right)^{1 / 2}
$$

By choosing $D, s, r$, and $N$ appropriately, we can find a $k^{2}$ from (A3) which also satisfies (A6), and then solve (A5) for $\alpha$ (we take the larger root for $\alpha$, so that $k_{c}^{2}$ is positive). This determines the point in $(N, D, r, s, \alpha)$ parameter space where mode (A6) is isolated. 


\section{ACKNOWLEDGMENTS}

This work (J.D.M.) was supported in part by Grant DMS-9003339 from the U.S. National Science Foundation, and (K.H.W.) by the Underlying Program of the U.K.A.E.A. M.R.M. acknowledges an Overseas Research Studentship (ORS) Award from the British Government and both M.R.M. and P.K.M. thank U.K.A.E.A. Harwell for their hospitality and support.

\section{REFERENCES}

1. J. D. Murray, Mathematical Biology. Springer-Verlag, Heidelberg ( 1989 ).

2. G. F. Oster and J. D. Murray, Pattern formation models and developmental constraints. $J$. Exp. Zool. 251, 186-202 (1989).

3. P. K. Maini, M. R. Myerscough, K. H. Winters, and J. D. Murray, Bifurcating spatially heterogeneous solutions in a chemotaxis model for biological pattern generation, Bull. Math. Biol.

4. J. D. Murray, A pattern formation mechanism and its application to mammalian coat markings. Lecture Notes in Biomath. 39, 360-399 (1979).

5. J. D. Murray, A pre-pattern formation mechanism for animal coat markings. $J$. Theor. Biol. 88, 161-199 (1981).

6. J. D. Murray, On pattern formation mechanisms for lepidopteran wing patterns and mammalian coat markings. Philos. Trans. Roy. Soc. London Ser. B 295, 473-496 (1981).

7. J. B. L. Bard, A model for generating aspects of zebra and other mammalian coat patterns. J. Theor. Biol. 93, 363-385 (1981).

8. J. D. Murray, P. K. Maini, and R. T. Tranquillo, Mechanochemical models for generating biological pattern and form in development. Phys. Rep. 171(2), 59-84 (1988).

9. J. D. Murray, Modelling the pattern formation mechanism in the formation of stripes on alligators. In Proc. IXth International Congress on Mathematical Physics, Swansea, pp. 208213 (1989).

10. J. D. Murray, D. C. Deeming, and M. J. W. Ferguson, Size dependent pigmentation pattern formation in embryos of Alligator mississipiensis: Time of initiation of pattern formation mechanism. Proc. Roy. Soc. London Ser. B239, 279-293 (1990).

11. M. Golubitsky and D. G. Schaeffer, Singularities and Groups in Bifurcation Theory, Part I. Springer-Verlag, New York/Berlin (1985).

12. A. D. Jepson and A. Spence, Singular points and their computation. In T. Küpper, H. D. Mittleman, and H. Weber (Eds.), Numerical Methods for Bifurcation Problems. Birkhäuser, Cambridge, MA (1984).

13. K. H. Winters, Th. Plesser, and K. A. Cliffe, The onset of convection in a finitc container due to surface tension and buoyancy. Physica 29D, 387-401 (1988).

14. G. Moore and A. Spence, The calculation of limit points of non-linear equations. SIAM J. Numer. Anal. 17, 567 (1980).

15. H. B. Keller, Numerical solutions of bifurcations and non-linear eigenvalue problems. In P. H. Rabinowitz (Ed.), Applications of Bifurcation Theory, pp. 359-384, Academic Press, New York (1977).

16. K. H. Winters, K. A. Cliffe, and C. P. Jackson, The prediction of instabilities using bifurcation theory. In R. W. Lewis, E. Hinton, P. Bettess, and B. A. Schrefler (Eds.), Numerical Methods for Transient and Coupled Problems, pp. 179-198 (1987). 